\title{
Mean Opinion Score Based Handover Protocol with Particle Swarm Optimization for Multi Objective Constraints
}

\author{
R.Thamil Pritha ${ }^{1}$, Dr. T. Menakadevi ${ }^{2}$ \\ Department of Electronics and Communication Engineering,Adhiyamaan College of Engineering,Hosur- \\ 635109 ,
}

\begin{abstract}
In the fast moving world, the wireless users expect a high data rate and rigid connectivity. For that the Next Generation Wireless Networks (NGWNs) are expected to provide high data rate and optimized quality of service to multimedia and real-time applications over the Internet Protocol (IP) network. To achieve these goals, handover plays a very critical role in maintaining the seamless connectivity when mobile terminals move across different cells or networks. In this paper, we propose a scheme for handoverprocess in an integrated scenario with Universal Mobile Telecommunication System (UMTS) and Worldwide Interoperability for Microwave Access (WiMAX) networks. The call quality is measured using Mean Opinion Score (MOS), as the major metric for handover optimization. The objective of this paper is to choose optimal MOS by using a multi objective Particle Swarm Optimization (PSO) algorithm for the selection of best base station. The proposed PSO based MOS handover scheme is compared with the traditional MOS-based handover scheme. The proposed work provides best base station selection with $20 \%$ reduction in delay as compared with the previous works. Similarly, the packet loss has been reduced to 50\%, call dropping probability has been decreased as $20 \%$ and handover probability is found to be reduced up to $30 \%$ as compared with the existing schemes. Also, the band width of the proposed system has been increased at 5\%. The numerical results are expected to demonstrate that our proposed scheme can maintain high call quality and reduce the probabilities for both handover dropping and call dropping. The proposed system is implemented and analyzed using MATLAB 7.10 with communication and mathematical tool boxes. TheHereditary Dominating Pair (HDP) and Code Domain Power (CDP) are plotted using MATLAB simulation.
\end{abstract}

Index Terms: Mean Opinion Score, Particle Swarm Optimization.

\section{Introduction}

The envisaged NGWNs integrate a number of different networks, such as Universal Mobile Telecommunications System (UMTS) and Worldwide Interoperability for Microwave Access (WiMAX) to provide a comprehensive and secure all IP based services to mobile terminals. Future mobile terminals will be equipped with multiple network interface cards, which enable the mobile users to connect to different networks and access any service anywhere and anytime. The traditional handover protocols based on RSS or cost functions have flaws and are not competitive enough to achieve satisfactory QOS. The mobile terminal has to scan continuously for the current and available networks signal strength. This scanning procedure utilizes wireless resources and also encounters with the wireless channel access delay. Mobile terminals with continuous scanning also consume more battery power, thereby resulting in energy inefficiency. Another problem is the fading signal which will give rise to the Ping-Pong effect, resulting in unnecessary handover [1].

With a growing number of moving users, it has become a necessity to guarantee the Quality of Service (QOS) for applications that demand more bandwidth, better network connectivity and seamless handover. Moreover, wireless networks are susceptible to delay, packet loss and poor call quality due to the low Signal to Interference and Noise Ratio (SINR). An efficient handover management scheme should be designed to achieve better call quality in NGWNs. In the existing handover schemes, a handover is generally triggered by either the detection of degradation in Received Signal Strength (RSS) or using other metrics such as measurement from network load, power consumption, user preference and available bandwidth [2].

\section{Related Work}

In the IEEE 802.21 standard [3], Media Independent Handover Functions (MIHFs) are defined to provide a generic link layer. The MIH framework provides a group of MIH functionalities that facilitate both mobile-initiated and network-initiated handovers. MIH provides a framework which exchanges the events, commands and information about QOS parameters, current link layer conditions and traffic load with different radio access technologies, which are used as input for takingdecision for handover.

Several authors had published a paper based on handover process in UMTS, WLAN and WiMAX technologies. These papers concentrate on bandwidth [4], SINR [5] or RSS [6] [7]. S. Yang et al [8] had proposed that, when roaming is carried out from WiMAX networks to Wi-Fi networks, it is reasonable to 
initialize handover process to Wi-Fi networks. The reason is Wi-Fi networks can provide high bandwidth and lower cost.However, they do not consider the handover probability. So when the handover is required,for the Wi-Fi network it increase the handover probability and affecting QOS. K .Yang had reported [5], a handover algorithmwhich is used toreceive SINR from various access networks as the handover criteria. There are different environmental and networking factors which causes variation in SINR. This results in increase in the handover probability and might cause unnecessary handover.

K .Lee and S. Kim [9] is based on forced termination of calls due to handover failure. The dropping of ahandover call isgenerally considered more serious than blocking of a new call. Therefore, a certain amount of bandwidth (also called guard channels) is exclusively reserved for handovers. This amount of bandwidth can be either fixed or adaptively controlled with respecttothecurrent traffic load. RSS and bandwidth are important factors but there are several other factors which might degrade the quality of voice signal. A usermight be just standing beside the base station and there might be sufficient bandwidth available, but the network to which the user is attached might not support VoIP call well, or there might be other network available which might provide better quality.

\section{Proposed Handover Protocol}

The IEEE 802.21 standard defines a media-independent handover (MIH) framework that can significantly improve seamless handover between heterogeneous network technologies. IEEE 802.21 facilitates the handover between different radio access technologies without call interruption, providing seamless connectivity for the mobile terminal, and improving the quality of service. MIH framework is based on a protocol stack implemented in all the devices involved in the handover, and provides a commoninterface for the link layer functions which is independent of radio access technologies. It consists of a MIH client which sits at user equipment end. MIH server resides in the core network. Handover decision for all the users in that zone is based on the information provided by MIH.

Fig.1 shows how the handover is triggered by the mobile terminal, whereas the decision on whether the handover will be finally performed and how the handover is performed are made at the RNC. The mobile terminal monitors the call quality and the current link state. If the mobile terminal detects the MOS value of the current ongoing call is below a predefined threshold, it sends a request to the RNC for handover. Once the RNC receives the request message, it will send a query to the candidate base stations. If there is another base station that can provide better service to the mobile terminal, handover will be immediately executed; otherwise the handover request is rejected.

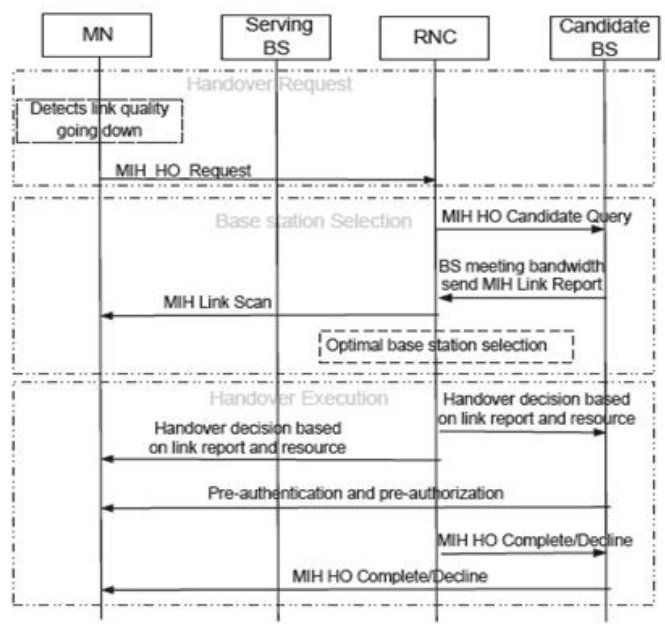

Fig.1: Handover Process

The major components include: MIH function (MIHF), which is a logical entity that provides abstract services to the higher layers through a media independent interface and obtains information from the lower layers through media specific interfaces.

It provides three types of services: (1) Media-Independent Event Service (MIES) for detecting and reporting changes in link layer properties; (2) Media-Independent Command Services (MICS) for local or remote MIH users to control link state; and (3) Media-Independent Information Service (MIIS) for providing information about neighboring network Service Access Points (SAPs), which defines both media independentand media-specific interfaces. It includes: (1) MIH SAP for high layers to control and monitor 
different links; (2) MIH LINK SAP for MIHF to control media-specific links; (3) MIH NET SAP to support the exchange of MIH information and messages with the remote MIHF.

To perform the MOS-based handover, our protocol needs the following information: the set of candidate base stations, theavailable bandwidth capacity at each candidate base station, the delay and packet loss rate for each wireless link between the mobile terminal to a candidate base station, and the MOS of the current connection. All these information can be obtained using the MIH functions provided in the IEEE 802.21 framework, as described below.

\section{PARTICLE SWARM OPTIMIZATION}

Particle swarm optimization is an algorithm to find the best solution for a problem. 'Particle' here refers to a candidate who is seeking for the best base station during the handover process. 'Swarm' refers to the solution for the handover process that is the selection of best base station in this work.

\subsection{PSO ALGORITHM STEPS}

PSO is initialized with a group of random particles (solutions)

$>$ Then searches for optima by updating generations

$>$ In every iteration, each particle is updated by following two "best" values.

$>$ The first one is the best solution (fitness) it has achieved so far. (The fitness value is also stored.) This value is called 'pbest'.

$>$ Another "best" value that is tracked by the particle swarm optimizer is the best value, obtained so far by any particle in the population. This best value is a global best and called 'gbest'.

$>$ When a particle takes part of the population as its topological neighbors, the best value is a local best and is called 'lbest'.

After finding the two best values, the particle updates its velocity and positions with following equation (a) and (b).

$v[]=v+c 1 * \operatorname{rand} *($ pbest []$-\operatorname{present}[])+c 2 * \operatorname{rand}() *($ gbest []$-\operatorname{present}[])$

present [] $=$ present [] $+v[]$

$v$ [] is the particle velocity, present [] is the current particle (solution). Pbest []and gbest [] are defined as stated before. rand ()is a random number between $(0,1)$.

The pseudo code of the procedure is as follows,

**For each particle

\{

Initialize particle

END

Do

For each particle

Calculate fitness value

\{

If the fitness value is better than the best fitness value 'pBest' in history

set current value as the new 'pBest'

$$
\text { \} }
$$

End

Choose the particle with the best fitness value of all the particles as the ' $\mathrm{gBest}$ '

For each particle

Calculate particle velocity according equation (a)

\}

Update particle position according equation (b)

\section{End}

While maximum iterations or minimum error criteria is not attained.

Fig. 2 shows how the search point is modified by using the PSO algorithm where $\mathrm{S}^{\mathrm{k}}$ is the current searching point, $\mathrm{S}^{\mathrm{k}+1}$ is the modified searching point, $\mathrm{V}^{\mathrm{k}}$ is the current velocity, $\mathrm{V}^{\mathrm{k}+1}$ is the modified velocity $\mathrm{V}_{\text {pbest }}$ velocity based on pbest, $\mathrm{V}_{\mathrm{gbest}}$ velocity based on gbest. 


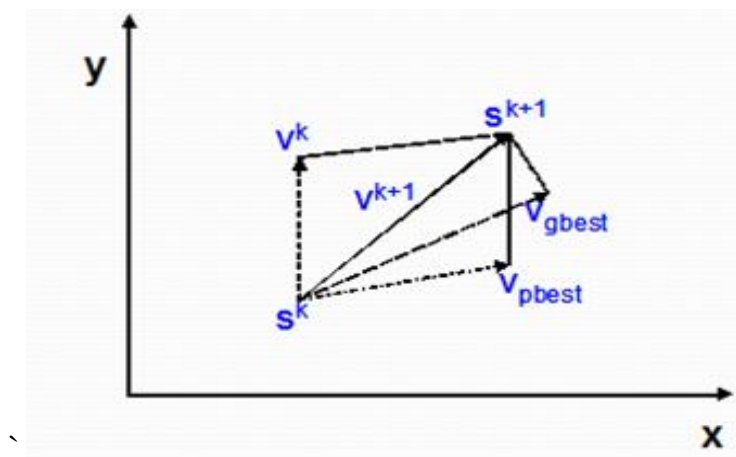

Fig.2: Search point through PSO

The flowchart in the fig. 3 indicates how the best optimal base station is selected by first Initializing the particle. Then for each particle calculate fitness value if the fitness value is better than the best fitness value 'pBest' in history, set current value as the new 'pBest'. At last choose the particle with the best fitness value of all the particles as the 'gBest' For each and every particle calculate particle velocity according equation (1), update particle position according equation (2) .

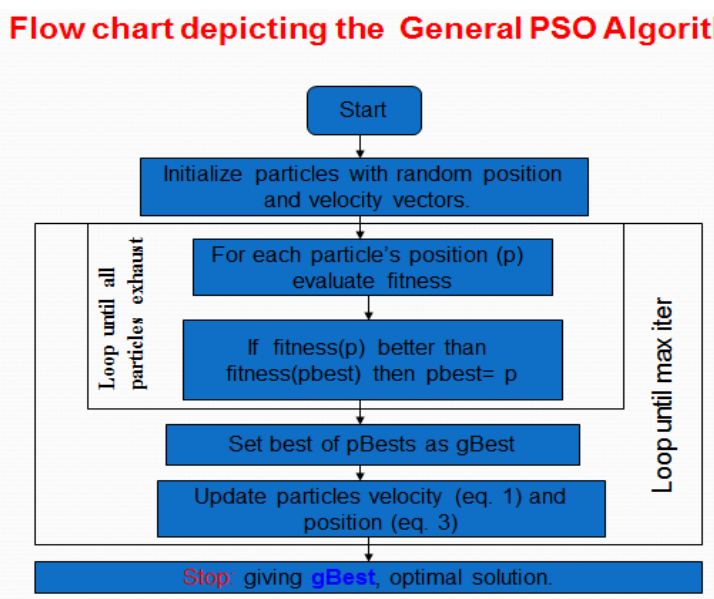

Fig.3: Flowchart of general PSO algorithm

\subsection{PSO PARAMETER CONTROL}

The major disadvantage of the Genetic Algorithm (GA) is the optimizationproblems: the representation of the solution and the fitness function. One of the advantages of PSO is that PSO take real numbers as particles. It is not like GA, which needs to change to binary encoding, or special genetic operators have to be used. For example, we try to find the solution for $\mathrm{f}(\mathrm{x})=\mathrm{x} 1^{\wedge} 2+\mathrm{x} 2^{\wedge} 2+\mathrm{x} 3^{\wedge} 2$, the particle can be set as $(\mathrm{x} 1, \mathrm{x} 2, \mathrm{x} 3)$, and fitness function is $\mathrm{f}(\mathrm{x})$. Then we can use the standard procedure to find the optimum. The searching is a repeat process, and the stop criteria are that the maximum iteration number is reached or the minimum error condition is satisfied. There are not many parameter need to be tuned in PSO.

\subsection{MEAN OPINION SCORE}

The MOS value of the current ongoing call is used to initiate the handover process and to compare with other potential connections. This information is directly available as there is an ongoing connection between the mobile terminaland the serving base station. In our protocol, the handover is triggered by the mobile terminal, whereas the decision on whether the handover will be finally performed and how the handover is performed are made at the RNC. The mobile terminal monitors the call quality and the current link state. If the mobile terminal detects the MOS value of the current ongoing call is below a predefined threshold, it sends a request to the RNC for handover. Once the RNC receives the request message, it will send a query to the candidate base stations. If there is another base station that can provide better service to the mobile terminal, handover will be immediately executed; otherwise the handover request is rejected. 
The Mean Opinion Score is calculated using the formula,

$M=1+0.035 R+7 * 10 e-6 R(R-60)(100-R)$

Where $R=94.2-I c-I d$

In which $I c$ and $I d$ are given as

$I c=g a m m a 1+(g a m m a 2 * \ln (1+\operatorname{gamma} 3 * p i, j))$

$I d=0.024 * d i, j+0.11(d i, j+0.11(d i, j-177.3) H(d i, j-177.3)$

After substituting the gamma values as gamma $1=0$, gamma $2=15$, gamma $3=30$ the equation is modified as

$$
I c=0+(30 * \log (1+(15 * 0.01))), I d=(0.024 * 5)+0.11 *(5-177.3) * 1
$$

The MOS value is finally obtained as,

$M=1+(0.035 * R)+(7 * 10 e-6 * R *(R-60) *(100-R))$

The RSS is calculated using the following function

$R S S=-62.5+26.5 \log 10(d)$

Where ' $\mathrm{d}$ ' is the distance between a mobile terminal and a base station. If mobile terminal detects the RSS value below the threshold, the mobile terminal scans for the available networks and handovers the call to the base station providing higher RSS. If the mobile terminal fails to find a better base station, the handover request is rejected, and the mobile terminal continues the call by connecting to the same base station.

\section{Results And Discussions}

The system under test was considered as a 3 network with heterogeneous elements. A simulation of hand over is triggered by considering the received signal below a threshold level, at such times a best network is chosen to continue the call based on various parameters like packet delay, bandwidth, data rate power consumption, and along with MOS. So this multi constraint objective function is tested over PSO and a resultant network is chosen.

In simulation,10 UMTS base stations and 4 WiMAX base stations in a $10000 \mathrm{~m} \_10000 \mathrm{~m}$ area. The mobile terminals are uniformly placed in the UMTS or WiMAX cells. Each of the UMTS or WiMAX cell has a base station. All the base stations are connected to the RNC where the handover algorithm is located. The diameter of a UMTS cell is configured to $2 \mathrm{~km}$, and the diameter of a WiMAX cell is configured to $3 \mathrm{~km}$. When a mobile user makes a VoIP call, the voice packets are carried from mobile terminal to the RNC through NodeB. Even though there are different codecs such as G.711, G.721, G.722, etc. We use G.711 since it has the least compression delay [10]. Each simulation is run for 10 minutes.

In simulation, a 2D random walk model to simulate the movement of the mobile terminals. Because some mobile terminals are believed to move in an unexpected way, random walk mobility model is proposed to mimic their movement behavior [11]. The random walk model is a stateless mobility process, where the information about the previous status is not used for the future decision. That is, the current parameter information is independent with its previous parameter information.

Fig.4 shows that the waveforms obtained when the first network is selected whereas Fig .4(a) shows the optimized MOS \& optimized MOS values of Handover probability vs. Time similarlyFig.4(b) shows the Call dropping probability\& Time and Fig.4 (c) shows which network has been selected and what is the MOS value for that network which is our assumption as set in the coding. In our coding the first network is assumed as 'WiMAX'. 


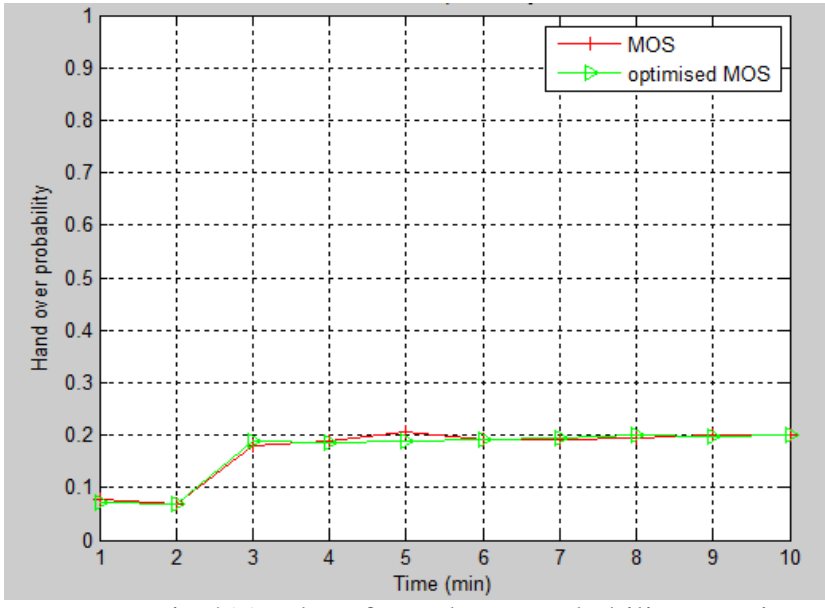

Fig.4(a): plot of Handover Probability vs. Time

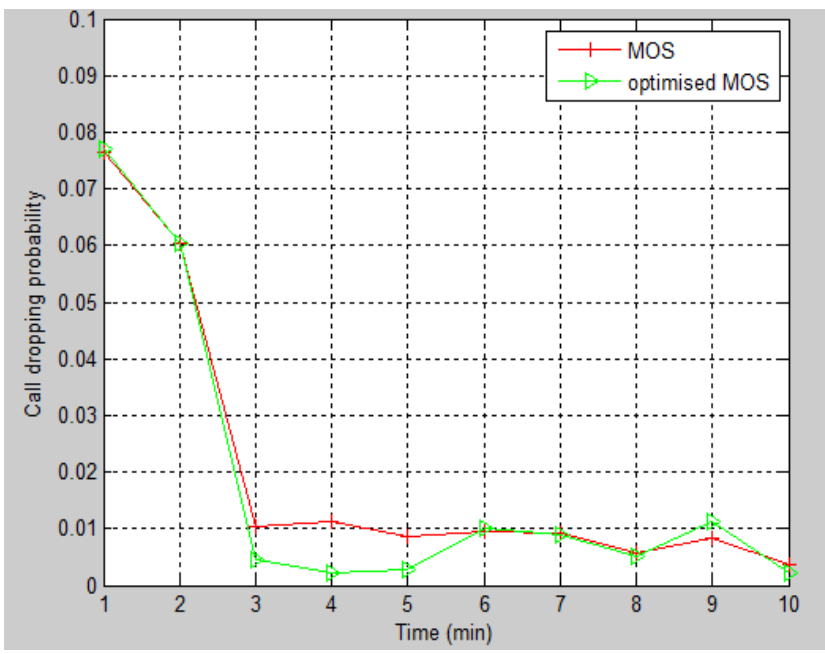

Fig.4(b): plot of Call dropping Probability vs. Time

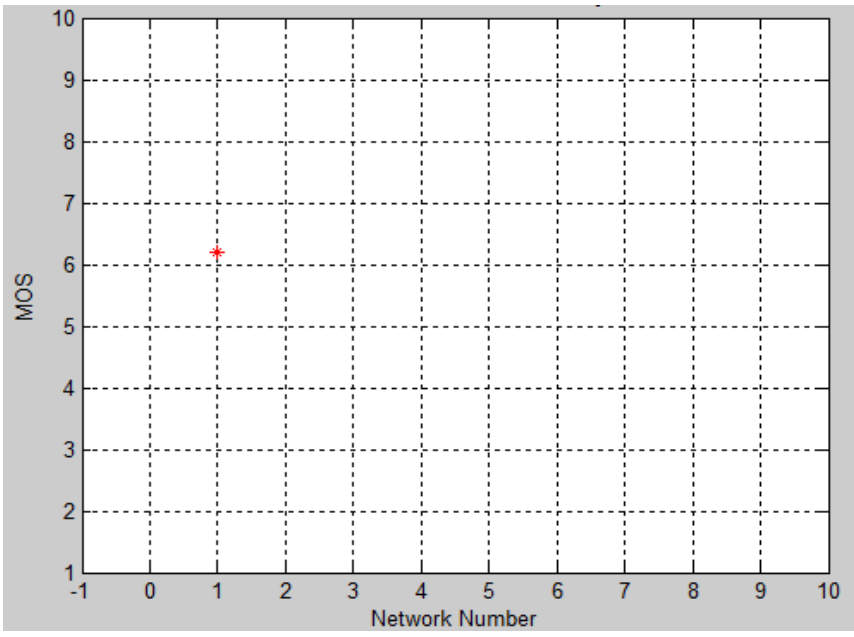

Fig.4 (c): plot of Network number vs. MOS 
Fig.5: Waveforms obtained when the second network is selected whereas Fig.5 (a) shows the optimized MOS \& optimized MOS values of Handover probability vs. Time similarlyFig.5 (b) shows the Call dropping probability \& Time and Fig.5 (c) shows which network has been selected and what is the MOS value for that network which is our assumption as set in the coding. In our coding the second network is assumed as ' $\mathrm{Wi}-\mathrm{Fi}$ '.

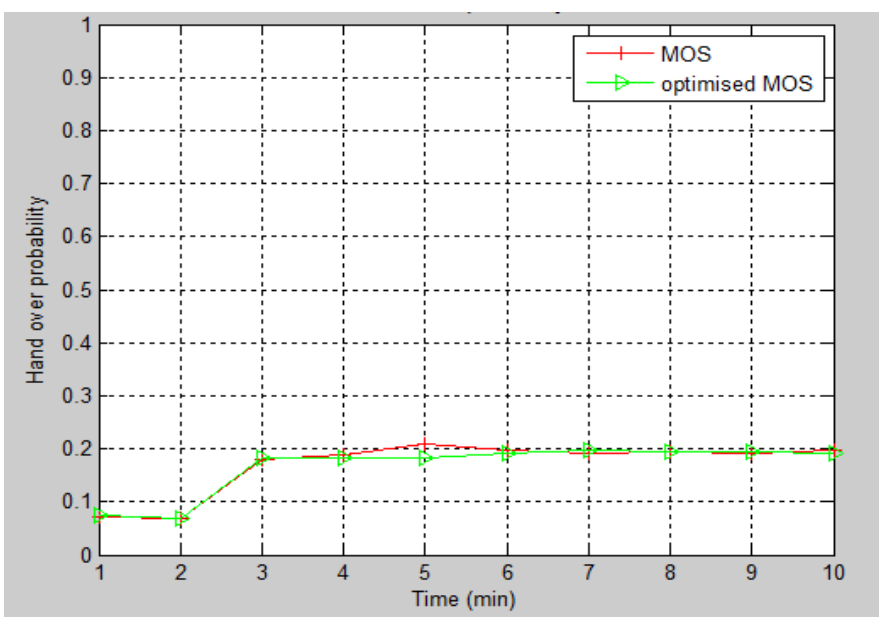

Fig.5 (a): plot of Handover Probability vs. Time

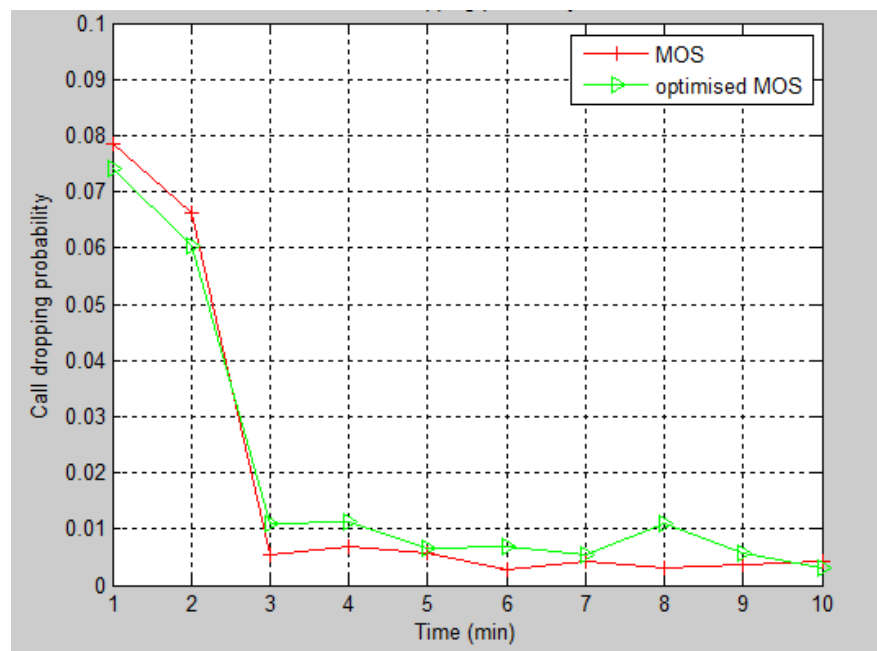

Fig.5 (b): plot of Call dropping Probability vs. Time

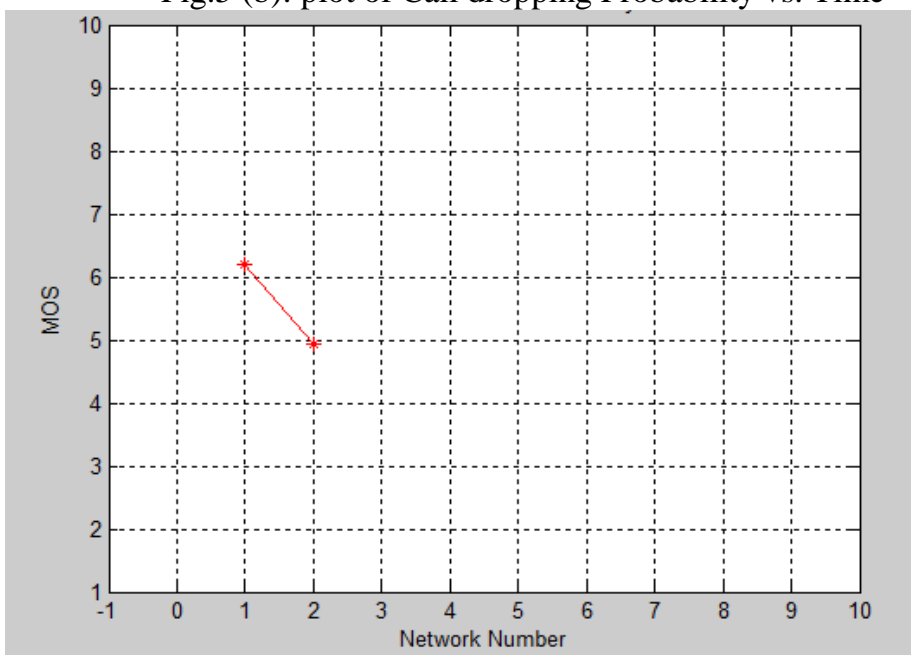

Fig.5 (c): plot of Network number vs. MOS 
Fig.6: Waveforms obtained when the Third network is selected whereas Fig.6 (a) shows the optimized MOS \& optimized MOS values of Handover probability vs. Time similarlyFig.6 (b) shows the Call dropping probability \& Time and Fig.6(c) shows which network has been selected and what is the MOS value for that network which is our assumption as set in the coding. In our coding the third network is assumed as 'UMTS'.

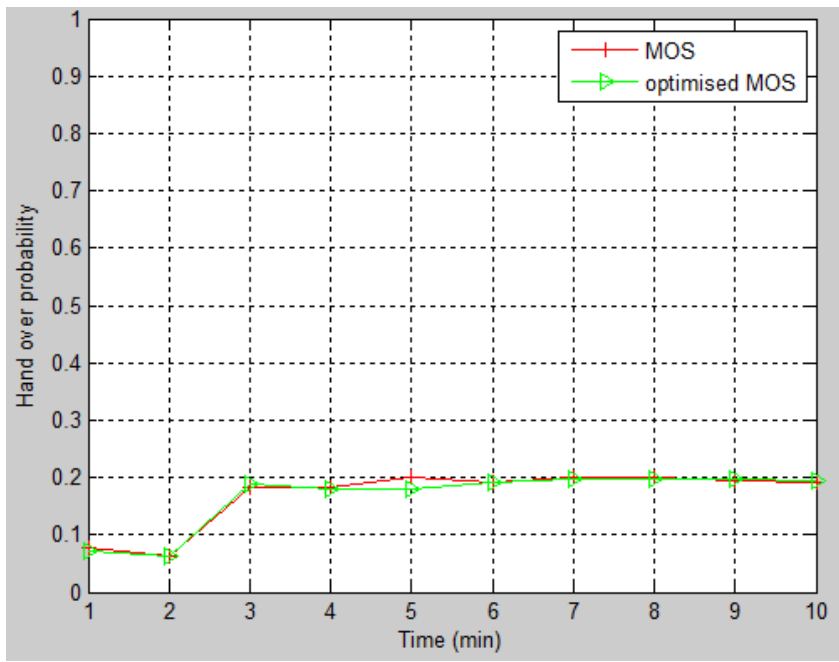

Fig.6 (a): plot of Handover Probability vs. Time

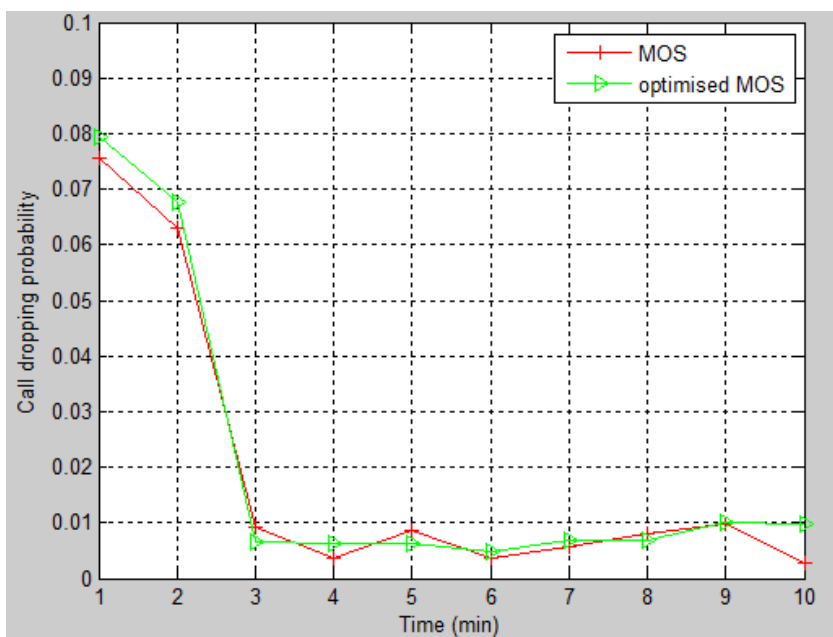

Fig.6 (b): plot of Call dropping Probability vs. Time

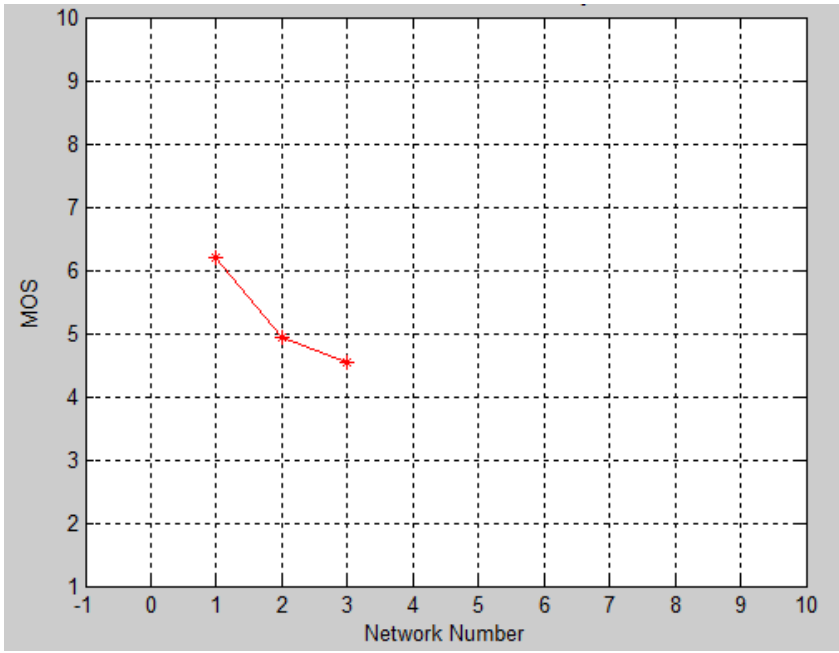

Fig.6 (c): plot of Network number vs. MOS 
TABLE -1: PERFORMANCE PARAMETER ANALYSIS FOR THE PROPOSED SCHEME.

\begin{tabular}{|l|l|l|c|c|c|l|}
\hline $\begin{array}{l}\text { NETWORKNU } \\
\text { MBER }\end{array}$ & \multicolumn{3}{|c|}{ HANDOVER PROBABILITY } & \multicolumn{2}{c|}{ CALL DROPPING PROBABILITY } \\
\hline & $\begin{array}{c}\text { TIME } \\
\text { Min) }\end{array}$ & MOS & OPTIMIZED MOS & $\begin{array}{l}\text { TIME } \\
\text { (Min) }\end{array}$ & MOS & $\begin{array}{l}\text { OPTIMIZED } \\
\text { MOS }\end{array}$ \\
\hline 1 & 5 & 0.20 & 0.019 & 4 & 0.011 & 0.003 \\
\hline 2 & 5 & 0.211 & 0.018 & 5 & 0.008 & 0.004 \\
\hline 3 & 5.5 & 0.20 & 0.019 & 3 & 0.010 & 0.005 \\
\hline
\end{tabular}

Table.1 shows the corresponding handover probability and the call dropping probability of various networks such as Wi-Max, Wi-Fi, UMTS, 2G. The handover probability at 5 minutes for the first network has the MOS value as 0.20 whereas for the optimized MOS value as 0.019 , for the second network MOS value as 0.211 whereas for the optimized MOS value as 0.018 , for the third network MOS value as 0.20 whereas for the optimized MOS value as0.019.

TABLE -2: COMPARISON BETWEEN PROPOSED SCHEME AND EXISTING SCHEME

\begin{tabular}{|cc|c|c|c|c|c|}
\hline AUTHORS & CDP & HOP & DELAY (Min) & PLP & BW (Mbs) \\
\hline Youssef Iraqi & $\mathbf{( 1 2})$ & 0.00276 & 0.05 & 0.1 & 0.05 & 1 \\
\hline SaravananKandasamy & $\mathbf{( 1 3 )}$ & 0.0025 & 0.21 & 0.09 & 0.04 & 1.1 \\
\hline Rujing Zhao & $\mathbf{( 1 4 )}$ & 0.0021 & 0.18 & 0.08 & 0.036 & 1.2 \\
\hline PROPOSED & 0.004 & 0.02 & 0.05 & 0.01 & 1.5 \\
\hline
\end{tabular}

Table.2 shows the comparison values of call dropping probability, handover probability, delay, packet loss probability and bandwidth. The proposed scheme shows the delay and probability values to be reduced whereas the bandwidth has been increased. The call dropping probability from various references has been compared and the proposed work provides a $20 \%$ reduction in delay. Similarly, the packet loss has been reduced to $50 \%$, and handover probability is found to be reduced up to $30 \%$. Also, the band width of the proposed system has been increased as $5 \%$.

\section{Conclusion}

The MOS is optimally chosen using a multi objective particle swarm optimization algorithm to choose the best base station based on delay, packet losses, bandwidth etc. The proposed PSO based MOS handover scheme with the traditional MOS-based handover scheme. The numerical results are expected to demonstrate that the proposed scheme can maintain high call quality and reduce the probabilities for both handover dropping and call dropping. The proposed work provides best base station selection and $20 \%$ reduction in delay as compared with the previous works. Similarly, the packet loss has been reduced to $50 \%$, call dropping probability has been decreased at $20 \%$ and handover probability is found to be $30 \%$ reduction as compared with the existing schemes. Also, the band width of the proposed system has been increased at 5\%.The system is implemented and analyzed using MATLAB software version 7.10 with communication and mathematical tool boxes. Hereditary Dominating Pair (HDP) and Code Domain Power (CDP) are plotted.

\section{References}

[1] X. Yan, Y. AhmetSekercioglu, and S. Narayanan, "A survey of vertical handover decision algorithms in Fourth Generation heterogeneous wireless networks," Computer Networks, vol. 54, no. 11, pp. 1848-1863, 2010.

[2] B. Chang and J. Chen, "Cross-layer-based adaptive vertical handoff with predictive RSS in heterogeneous wireless networks," IEEE Transactions on Vehicular Technology, vol. 57, no. 6, pp. 3679-3692, 2008.

[3] "IEEE Standard for Local and metropolitan area networks - Part 21: Media Independent Handover Services,” IEEE 802.21-2008.

[4] C. Oliveira, J. Kim, and T. Suda, "An adaptive bandwidth reservation scheme for high-speed multimedia wireless networks," IEEE Journal on Selected Areas in Communications,, vol. 16, no. 6, pp. 858-874, 1998.

[5] K. Yang, I. Gondal, B. Qiu, and L. Dooley, "Combined SINR based vertical handoff algorithm for next generation heterogeneous wireless networks," in IEEE Global Telecommunications Conference, 2007 (GLOBECOM' 07)., pp. $4483-4487$.

[6] S. Kunarak and R. Suleesathira, "Predictive RSS with fuzzy logic based vertical handoff algorithm in heterogeneous wireless networks," in IEEE International Symposium on Communications and Information Technologies (ISCIT), 2010, pp. 1235-1240.

[7] B. Chang, J. Chen, C. Hsieh, and Y. Liang, "Markov decision process-based adaptive vertical handoff with RSS prediction in heterogeneous wireless networks," in IEEE Wireless Communications and Networking Conference, 2009. WCNC 2009. 2009, pp. $1-6$.

[8] S. Yang, J. Wu, and Y. ROC, "Handoff management schemes across hybrid WiMAX and Wi-Fi networks," in IEEE TENCON, 2007.

[9] K. Lee and S. Kim, "Optimization for adaptive bandwidth reservation in wireless multimedia networks," Computer networks, vol. 38 , no. 5, pp. 631-643, 2002. 
[10] R. Cole and J. Rosenbluth, "Voice over IP performance monitoring," ACM SIGCOMM Computer Communication Review, vol. 31, no. 2, pp. 9-24, 2001.

[11] F. Bai and A. Helmy, "A survey of mobility models," Wireless Adhoc Networks.University of Southern California, USA, vol. 206, 2004.

[12] Youssef Iraqi, RaoufBoutaba,"Handoff and Call Dropping Probabilities in Wireless Cellular Networks", International Conference on Wireless Networks, Communications and Mobile Computing, 2005.

[13] SaravananKandasamy, Prihandoko, BorhanuddinMd.Ali, V. Prakash, and RatnaKalos,'Improved Handoff Call Dropping Probability (HCDP) in Adaptive Quality of Service (AdQoS) in Multimedia Wireless Networks Using Hierarchical Cellular Approach", 4-National Conference on Telecommunication Technology Proceedings, Shah Alam, Malaysia.

[14] Rujing Zhao, Xiangming Wen, Dongming Su, Wei Zheng," Call Dropping Probability of Next Generation Wireless Cellular Networkswith Mobile Relay Station", Second International Conference on Future Networks 2010. 